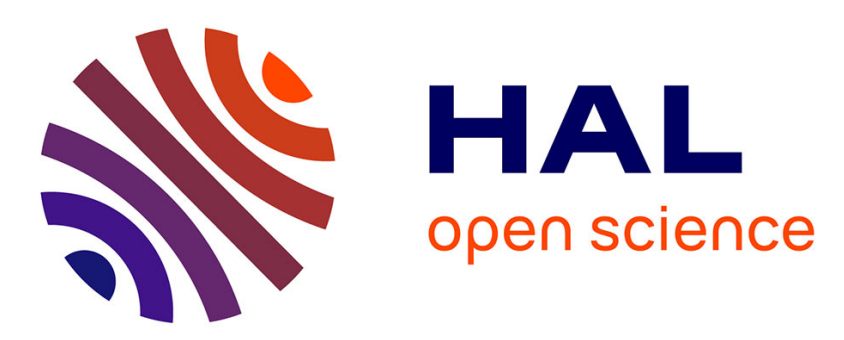

\title{
Collaborative Estimation of Gradient Direction by a Formation of AUVs under Communication Constraints
}

Lara Briñon Arranz, Alexandre Seuret, Carlos Canudas de Wit

\section{To cite this version:}

Lara Briñon Arranz, Alexandre Seuret, Carlos Canudas de Wit. Collaborative Estimation of Gradient Direction by a Formation of AUVs under Communication Constraints. CDC 2011 - ECC 2011 - 50th IEEE Conference on Decision and Control and European Control Conference, Dec 2011, Orlando, Floride, United States. pp.6. hal-00619482v2

\section{HAL Id: hal-00619482 \\ https://hal.science/hal-00619482v2}

Submitted on 6 Sep 2011

HAL is a multi-disciplinary open access archive for the deposit and dissemination of scientific research documents, whether they are published or not. The documents may come from teaching and research institutions in France or abroad, or from public or private research centers.
L'archive ouverte pluridisciplinaire HAL, est destinée au dépôt et à la diffusion de documents scientifiques de niveau recherche, publiés ou non, émanant des établissements d'enseignement et de recherche français ou étrangers, des laboratoires publics ou privés. 


\title{
Collaborative Estimation of Gradient Direction by a Formation of AUVs under Communication Constraints
}

\author{
Lara Briñón-Arranz, Alexandre Seuret and Carlos Canudas-de-Wit
}

\begin{abstract}
This work deals with the source-seeking problem in which the task is to locate the source of some signal using a fleet of Autonomous Underwater Vehicles (AUVs). The present paper proposes a distributed solution in which a group of vehicles uniformly distributed in a fixed circular formation, estimates the gradient direction of the signal propagation. The distributed algorithm takes into account the communication constraints and depends on direct signal measurements. Our approach is based on the previous results in formation control to stabilize the fleet in a circular formation with time-varying center and in a collaborative source-seeking algorithm. The results are supported through computer simulations.
\end{abstract}

\section{INTRODUCTION}

Cooperative control problems and multi-agent systems have received much attention in recent years. The field includes consensus algorithms for multi-agent systems [1], [2], flocking [3], distributed sensor networks [4], [5], and autonomous systems as underwater and unmanned air vehicles (AUVs and UAVs) [6], [7]. Cooperative formation control and motion coordination have been extensively studied, see [8], [9], [10], [11], among many others. Control laws have been provided to make a fleet of agents (vehicles) obtain circular and parallel formations [6], [12]. Many extensions based on these works have been developed: threedimensional formation control [13], [14], planar circular formation control in a flow-field [15], and stabilization of a fleet to other closed forms [16], [17].

In [18], a new control law is proposed to translate a circular formation following a desired external reference trajectory of its center. Designing a collaborative reference to move the formation is a first step to achieve the source location of some signal. The source could be a point of chemical contamination and the signal would be that chemical's concentration in the environment, for example. The objective of the source- seeking problem is to obtain the direction to steer the formation towards the source by a cooperative algorithm using the concentration measurements of the agents.

There exist many different approaches to resolve the source-seeking problem in the literature. The extremum seeking problem is solved under different hypotheses using

Lara Briñón-Arranz is with INRIA Rhône-Alpes, NeCS Team, Grenoble, France lara.brinon-arranz@inrialpes.fr

Alexandre Seuret and Carlos Canudas-de-Wit are with the Department of Automatic Control, GIPSA-Lab, CNRS, NeCS Team, Grenoble, France $\{a l e x a n d r e$. seuret, carlos.canudas-de-wit\} @gipsa-lab.grenoble-inp.fr

This work was supported by EU STREP project FeedNetBack FP7-ICT2007-2. a single nonholonomic vehicle, [19], [20]. In this case all the measurements would come from a single vehicle as it changes position over time. Another strategy consists in approximating the gradient value of the signal using concentration measurements of multiple vehicles at different locations [4]. Some collaborative methods have been proposed, based on distributed estimation of the concentration plume [21], [22]. In theses cases, the function signal is estimated or approximated and the source localization becomes a distributed optimization problem.

The work presented in [23] deals with a collaborative multi-agent algorithm to solve the source seeking problem using only direct signal measurements by a circular formation of agents. This new approach considers all-toall communication (i.e., every agent talks to every other agent) between the vehicles and the control law needs the information of all the agents to converge. Our objective now is to design a distributed algorithm based on this previous one but considering restricted communication. The present paper addresses an other possible solution to the source localisation problem. We show how a group of vehicles uniformly distributed in a circular formation, is able to approximate the gradient direction of the signal propagation to steer the formation towards the source location. In order to achieve this objective under limited communication, each agent estimates its own direction based on its neighbors' concentration measurements. We include a consensus algorithm to converge to the same desired gradient direction. In literature there are some results in the field of consensus filters and sensor fusion [2], [5] which deal with the consensus problem of a sensor network measuring a signal corrupted by noise. These approaches could be applied to reduce the noise propagation and to cooperative exploration missions.

This paper is organized as follows. First, Section II recalls previous work on translation control of a circular formation. Section III presents a result on gradient approximation and the problem formulation. In Section IV we propose a first algorithm that combines standard consensus tools, and show its limitations both by theoretical convergence analysis and simulations. Then, Section V presents an improved estimation algorithm based on averaging. Conclusions and future works are presented in Section VI.

Notation. Let $\mathcal{G}=(V, E)$ be an undirected graph with an adjacency matrix $\mathcal{A}=\left[a_{k j}\right]$ that specifies the communication topology of the multi-agent system: $a_{k j}=1$ if agents $k$ and $j$ $\in V$ communicate, else $a_{k j}=0$. The set of nodes (agents) is denoted by $V=\{1, \ldots, N\}$. Let $\mathcal{N}_{k}=\left\{k \in V: a_{k j} \neq 0\right\}$ 
the set of neighbors of agent $k$ and $\mathcal{J}_{k}=\mathcal{N}_{k} \cup\{k\}$. The Laplacian matrix $\mathbf{L}$ of graph $\mathcal{G}$ is defined as $\mathbf{L}=\Delta-\mathcal{A}$ where $\Delta$ is the diagonal matrix which contains the degree of each agent, i.e., $\Delta_{k k}=d_{k}=\sum_{j} a_{k j}$. In the sequel, $\otimes$ denotes the Kronecker product and, for simplicity, we define $\mathbf{M}_{2}=\mathbf{M} \otimes \mathbf{I}_{2}$ where $\mathbf{M}$ is a square matrix and $\mathbf{I}_{N}$ is the identity matrix of order $N$. Moreover, $\mathbf{R}_{\frac{\pi}{2}} \in \mathbb{R}^{2 \times 2}$ denotes the rotation matrix counterclockwise through an angle $\frac{\pi}{2}$.

\section{BACKGROUND}

The algorithm to estimate the gradient direction of a signal distribution presented in this paper builds on the previous result on formation control from [18]. Consider a group of $N$ agents modeled as kinematic unicycle vehicles of the following form, for each vehicle $k$ :

$$
\begin{gathered}
\dot{x}_{k}=v_{k} \cos \theta_{k} \\
\dot{y}_{k}=v_{k} \sin \theta_{k}
\end{gathered}
$$

where $r_{k}=\left(x_{k}, y_{k}\right)^{T}$ is the position vector, $\theta_{k}$ is the heading angle, and the control inputs are the vehicle's forward velocity $v_{k}>0$ and turning rate $\dot{\theta}_{k}$, see [18]. With appropriate limits on the control inputs, this model can provide a reasonable approximation for many air and underwater vehicles.

Stabilization of a fleet of $N$ agents to a circular formation around its center of mass was developed in [6]. In [18] a control law which asymptotically stabilizes the vehicles to a circular formation around a dynamic center point $c(t)=$ $\left(c_{x}, c_{y}\right)^{T}$ with a uniform distribution (i.e., with the agents evenly separated on the circle by $2 \pi / N$ radians each) is presented. The center of the formation $c(t)$ is an external reference known for all the agents. With a fixed radius $R>$ 0 , desired rotational velocity $\omega_{0}$ and the control parameters $\kappa>0$ and $K$, the control law of [18] is given as:

$$
\begin{aligned}
v_{k} & =\left\|R\left|\omega_{0}\right|\left(\cos \psi_{k}, \sin \psi_{k}\right)^{T}+\dot{c}\right\| \\
\dot{\theta}_{k} & =\left(1-\frac{\dot{r}_{k}^{T} \dot{c}}{v_{k}^{2}}\right) \dot{\psi}_{k}-\frac{\dot{r}_{k}^{T} \mathbf{R}_{\frac{\pi}{2}} \ddot{c}}{v_{k}^{2}} \\
\dot{\psi}_{k} & =\omega_{0}\left(1+\kappa R\left|\omega_{0}\right|\left(\cos \psi_{k}, \sin \psi_{k}\right)\left(r_{k}-c\right)\right)-\frac{\partial U}{\partial \psi_{k}} \\
U(\psi) & =-\frac{K}{N} \sum_{m=1}^{\lfloor N / 2\rfloor} \frac{1}{2 m^{2}} \mathbf{a}_{m}^{T} \mathbf{L}_{2} \mathbf{a}_{m}
\end{aligned}
$$

where $\lfloor N / 2\rfloor$ is the largest integer less than or equal to $N / 2$, $\mathbf{a}_{m}=\left(\cos m \psi_{1}, \sin m \psi_{1}, \ldots, \cos m \psi_{N}, \sin m \psi_{N}\right), \mathbf{L}$ is the Laplacian matrix associated with the communication network of the vehicles, and $\psi_{k}(t)$ is an inner state of the dynamic controller, and initialized to

$$
\psi_{k}(0)=\arctan \frac{\dot{y}_{k}(0)-\dot{c}_{y}(0)}{\dot{x}_{k}(0)-\dot{c}_{x}(0)}+\epsilon_{k} \pi
$$

where $\epsilon_{k}=0$ if $\dot{x}_{k}(0)-\dot{c}_{x}(0)>0$ and $\epsilon_{k}=1$ otherwise.

Assuming that $c(t)$ is twice differentiable, has bounded first and second time-derivatives, and satisfies $\sup _{t \geq 0}|\dot{c}(t)|<$ $R\left|\omega_{0}\right|$, then the control law above drives the vehicles to trajectories that lie on the circle with radius $R$ and whose center

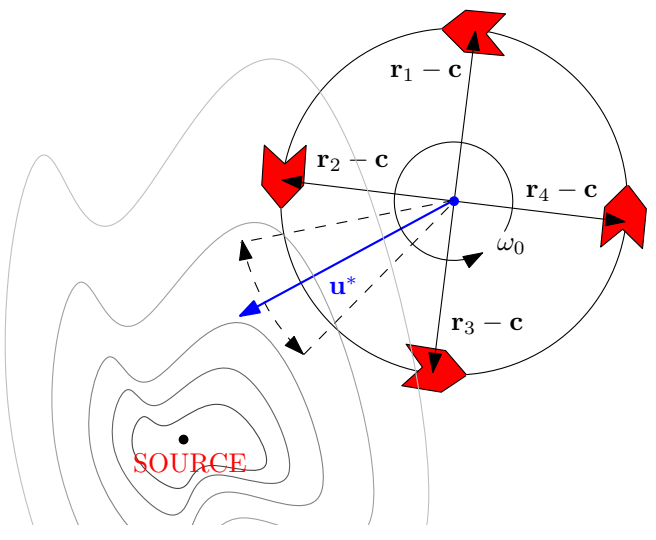

Fig. 1. Problem formulation

follows the time-varying external reference $c(t)$. Moreover, if $K>0$ and a geometric ring communication graph (i.e,. each agent is linked to its two closest neighbors on the circle) is assumed, then the vehicles will be uniformly distributed on that circle.

Consider now the stable circular formation described by a center point $c$, a radius $R$ and an angle $\phi$ which is linearly increasing with time (i.e., $\phi=\omega_{0} t$ for some angular speed $\omega_{0}>0$. Therefore, the position of each agent $k$ is given by the following equation:

$$
\begin{aligned}
& x_{k}=c_{x}+R \cos \left(\phi+k \frac{2 \pi}{N}\right) \\
& y_{k}=c_{y}+R \sin \left(\phi+k \frac{2 \pi}{N}\right)
\end{aligned}
$$

This equation describes a formation where the agents are uniformly distributed on a circle of radius $R$. In the context of source-seeking problem, the objective is that the center of the formation $c(t)$ follows a trajectory which converges to the maximum of a signal, that is usually its source. Using this previous work on formation control, a first result in collaborative source-seeking is accomplished in [23]. The authors consider here a stable circular formation of $N$ mobile agents in the plane. The agents are stabilized by the previous control law (2). The authors provide an outer- loop control that steers the formation by determining $\dot{c}(t)$ in a collaborative way. This control law allows the formation to move such that its center converges to the source location, if the signal distribution decreases around the source in such a way that level sets are circles or ellipses centered on source location. The main constraint of the algorithm of [23] is the all-to-all communication assumption.

\section{PROBLEM ForMULATION}

The objective of this work, is to estimate the gradient direction of the source distribution based on the concentration measurements obtained by a circular formation of agents, taking into account the communication constraints. This estimated direction will be the reference velocity of the 
formation center in order to steer the group of agents to the source location.

Consider a fleet of $N$ vehicles uniformly distributed along a circular formation. The position of each agent $k$ is described by equation (3). Our approach considers that the circular formation is not moving. Only the estimation of gradient direction is addressed here.

Assumption 1 In the sequel, we suppose that the center of the circular formation $c$ is fixed and known to all the agents.

The distribution of the signal strength in the environment will be described by an unknown positive spatial mapping $\rho$ : $\mathbb{R}^{2} \rightarrow \mathbb{R}^{+}$, and so agent $k$ measures the signal strength at its position as $\rho\left(r_{k}\right)$. Our approach deals with the approximation of the gradient direction of this signal distribution $\rho$ by a circular formation of agents at a given location $c$, see Fig. 1 .

\section{A. Gradient Approximation}

Consider a fleet of agents given by (3) taking measurements of a signal distribution $\rho$. Let $\nabla \rho(c)=$ $\left(\nabla_{x} \rho(c), \nabla_{y} \rho(c)\right)$ denote the gradient of function $\rho$ in the center of the circular formation. The following lemma is proposed:

Lemma 1 Let $\rho$ be a bounded function and $\rho\left(r_{k}\right)$ the measure obtained by agent $k$ where $r_{k}$ is its position vector given by (3). If Assumption 1 is satisfied and the agents are uniformly distributed along the circle centered at c, then:

(i) Considering a fleet of $N>2$ agents the following equation is satisfied:

$$
\frac{1}{N} \sum_{k=1}^{N} \rho\left(r_{k}\right)\left(r_{k}-c\right)=\frac{R^{2}}{2} \nabla \rho(c)^{T}+o\left(R^{2}\right)
$$

(ii) Considering a limitless number of agents along the circular formation $(N \rightarrow \infty)$ the following equation is satisfied:

$$
\frac{1}{2 \pi} \int_{0}^{2 \pi} \rho\left(r_{k}\right)\left(r_{k}-c\right) d \phi=\frac{R^{2}}{2} \nabla \rho(c)^{T}+o\left(R^{2}\right)
$$

Proof: In both cases (i) and (ii) the uniform distribution of the agents along a fixed circle is assumed, then $\sum_{k=1}^{N}\left(r_{k}-\right.$ $c)=0$ and $\int_{0}^{2 \pi}\left(r_{k}-c\right) d \phi=0$ respectively. By definition of gradient of the function $\rho$ at a fixed location $c$ the following equation holds:

$$
\rho\left(r_{k}\right)-\rho(c)=\nabla \rho(c)\left(r_{k}-c\right)+o(R)
$$

Multiplying this previous equation by the relative vector $\left(r_{k}-c\right)$ and summing over $k=1, \ldots, N$, it yields:

$\frac{1}{N} \sum_{k=1}^{N} \rho\left(r_{k}\right)\left(r_{k}-c\right)=\frac{1}{N} \sum_{k=1}^{N}\left[\nabla \rho(c)\left(r_{k}-c\right)\right]\left(r_{k}-c\right)+o\left(R^{2}\right)$

Analyzing in terms of components and using (3) to express the position of the agents $r_{k}$, the right-hand side of the previous equation is given by:

$$
\frac{R^{2}}{N} \sum_{k=1}^{N}\left(\begin{array}{c}
\nabla_{x} \rho(c) \cos ^{2} \phi_{k}+\nabla_{y} \rho(c) \cos \phi_{k} \sin \phi_{k} \\
\nabla_{x} \rho(c) \sin \phi_{k} \cos \phi_{k}+\nabla_{y} \rho(c) \sin ^{2} \phi_{k}
\end{array}\right)
$$

where $\phi_{k}=\phi+k \frac{2 \pi}{N}$. Thanks to the uniform distribution if $N>2$ then $\sum_{k=1}^{N} \cos \left(2 k \frac{2 \pi}{N}\right)=0$ (the same equality also holds for the sinus) and trigonometric properties ensure that:

$$
\frac{1}{N} \sum_{k=1}^{N}\left[\nabla \rho(c)\left(r_{k}-c\right)\right]\left(r_{k}-c\right)=\frac{R^{2}}{2} \nabla \rho(c)^{T}
$$

Thus, the equality (4) is satisfied.

A similar analysis can be applied to second case (ii). Using trigonometric properties, integrating along the circle (in the interval $[0,2 \pi])$ and thanks to the equality $\int_{0}^{2 \pi} \cos \phi d \phi=$ 0 (the same equality also holds for $\sin \phi$ ) we obtain the following equation:

$$
\frac{1}{2 \pi} \int_{0}^{2 \pi}\left[\nabla \rho(c)\left(r_{k}-c\right)\right]\left(r_{k}-c\right) d \phi=\frac{R^{2}}{2} \nabla \rho(c)^{T}
$$

and equality (5) is straightforwardly obtained.

This result provides an approximation of gradient of the signal distribution at the center of the circular formation.

\section{B. Problem Formulation}

The objective of this paper is to develop a distributed algorithm to estimate the gradient direction of the signal distribution $\rho$ at the center of a circular formation of agents.

The communication constraints are taken into account through a communication graph $\mathcal{G}$. Due to these communication restrictions each agent estimates its own gradient direction $z_{k}$ using the information of its neighbors according to the communication topology. The objective is to make all estimated directions $z_{k}$ converge to the mean direction defined as:

$$
u^{*}=\frac{1}{N} \sum_{k=1}^{N} u_{k} ; \quad u_{k}=\rho_{k}\left(r_{k}-c\right)
$$

where $u_{k}$ is the relative position vector of agent $k$ weighted by its concentration measurement $\rho_{k}=\rho\left(r_{k}\right)$. Thanks to Lemma 1 (i), this mean vector $u^{*}$ approximates the gradient direction of the signal distribution at the center of the formation $c$. A consensus algorithm is implemented to obtain the same estimated gradient direction of the signal distribution for all the agents.

\section{Collaborative Estimation of GRAdient DIRECTION}

\section{A. Consensus algorithm}

Consensus filters for sensor networks are developed in [2]. In this work, the authors consider a sensor network of size $N$ with information flow (communication graph) $\mathcal{G}$. Each sensor $k$ measures the same signal that is corrupted by noise. Based on this approach for sensor networks, we propose the following consensus algorithm for the multi-agents system 
to estimate the gradient direction of the signal propagation by a fixed circular formation in a collaborative way:

$$
\dot{z}_{k}=\kappa \sum_{j \in \mathcal{N}_{k}} a_{k j}\left(z_{j}-z_{k}\right)+\sum_{j \in \mathcal{J}_{k}} a_{k j}\left(u_{j}-z_{k}\right)
$$

where $\kappa>0$ is a control parameter which is introduced to make the algorithm more flexible. The consensus variable is the vector $z_{k} \in \mathbb{R}^{2}$ which represents the estimated gradient direction by agent $k$. The input $u_{k}=\rho_{k}\left(r_{k}-c\right) \in \mathbb{R}^{2}$, depends on the concentration measurements and the position of the agent in the formation. Therefore, the input of the algorithm is not the same signal corrupted by noise, but a different vector for each agent. This is the main difference with respect to the consensus filter algorithm.

Using the Laplacian matrix of the communication topology of the multi-agents system the previous equation can be rewritten in a matrix way:

$$
\begin{aligned}
\dot{\mathbf{z}} & =-\kappa \mathbf{L} \otimes \mathbf{I}_{2} \mathbf{z}+\mathbf{I}_{N} \otimes \mathbf{I}_{2}(\mathbf{u}-\mathbf{z})+\mathcal{A} \otimes \mathbf{I}_{2} \mathbf{u}-\Delta \otimes \mathbf{I}_{2} \mathbf{z} \\
& =-\left(\mathbf{I}_{N}+\Delta+\kappa \mathbf{L}\right)_{2} \mathbf{z}+\left(\mathbf{I}_{N}+\mathcal{A}\right)_{2} \mathbf{u}
\end{aligned}
$$

where $\mathbf{z}=\left(z_{1}^{T}, z_{2}^{T}, \ldots, z_{N}^{T}\right)^{T}$ and $\mathbf{u}=\left(u_{1}^{T}, u_{2}^{T}, \ldots, u_{N}^{T}\right)^{T}$ are vectors of dimension $2 N$, and $\mathbf{I}_{N}$ the identity matrix of order $N$. Let $\mathbf{A}_{\kappa}=\left(\mathbf{I}_{N}+\Delta+\kappa \mathbf{L}\right)_{2}$, and $\mathbf{B}=\left(\mathbf{I}_{N}+\mathcal{A}\right)_{2}$. Note that by definition, $\mathbf{A}_{\kappa}$ is a positive definite matrix. Then, the previous equation becomes:

$$
\dot{\mathbf{z}}=-\mathbf{A}_{\kappa} \mathbf{z}+\mathbf{B u}
$$

Consider the vector of dimension $2 N, \mathbf{u}^{*}=\mathbf{1} \otimes u^{*}$, where $\mathbf{1}=(1, \ldots, 1)^{T} \in \mathbb{R}^{N}$ is the vector of ones that is always a right eigenvector of $\mathbf{L}$ corresponding to the eigenvalue 0 . Then the error equation is $\eta=\mathbf{z}-\mathbf{u}^{*}$. Using (8), the dynamics of the error can be written as:

$$
\dot{\eta}=-\mathbf{A}_{\kappa} \eta+\mathbf{B}\left(\mathbf{u}-\mathbf{u}^{*}\right)-\dot{\mathbf{u}}^{*}
$$

The stability of this algorithm is analyzed using the Lyapunov function $V=\frac{1}{2} \eta^{T} \mathbf{A}_{\kappa} \eta$. Differentiating this function we obtain:

$$
\dot{V}=-\eta^{T} \mathbf{A}_{\kappa}^{T} \mathbf{A}_{\kappa} \eta+\left(\mathbf{u}-\mathbf{u}^{*}\right)^{T} \mathbf{B}^{T} \mathbf{A}_{\kappa} \eta-\dot{\mathbf{u}}^{* T} \mathbf{A}_{\kappa} \eta
$$

Let $\left\|\dot{u}^{*}\right\| \leq \nu$, due to the soft variation of the concentration levels of the signal distribution considered here, then

$$
\begin{aligned}
\dot{V} \leq & -\lambda_{\min }^{2}\left(\mathbf{A}_{\kappa}\right)\|\eta\|^{2}+\nu \sqrt{N}\left(1+d_{\max }\right)\|\eta\| \\
& +\left\|\left(\mathbf{u}-\mathbf{u}^{*}\right)^{T} \mathbf{B}^{T} \mathbf{A}_{\kappa} \eta\right\|
\end{aligned}
$$

This is because

$$
\begin{aligned}
\dot{\mathbf{u}}^{* T} \mathbf{A}_{\kappa} & =\mathbf{1}^{T} \otimes \dot{u}^{* T}\left(\mathbf{I}_{N}+\Delta+\kappa \mathbf{L}\right)_{2} \\
& =\left(1+d_{1}, \ldots, 1+d_{N}\right) \otimes \dot{u}^{* T}
\end{aligned}
$$

and thus

$$
\left\|\dot{\mathbf{u}}^{* T} \mathbf{A}_{\kappa}\right\| \leq\left\|\dot{u}^{*}\right\| \sqrt{2 \sum_{k=1}^{N}\left(1+d_{k}\right)^{2}} \leq \nu \sqrt{N}\left(1+d_{\max }\right)
$$

It is plausible to assume that a bound on maximal signal concentration is known from the problem setting. Therefore, $\left\|\left(\mathbf{u}-\mathbf{u}^{*}\right)\right\| \leq \alpha$ where $\alpha$ depends on the radius of the circular formation and on the greatest concentration measurement obtained by the agents. For simplicity, let $\gamma$ be a bound of the following matrix norm $\left\|\mathbf{B}^{T} \mathbf{A}_{\kappa}\right\| \leq \gamma$. Taking these considerations into account the following equation holds:

$$
\left\|\left(\mathbf{u}-\mathbf{u}^{*}\right)^{T} \mathbf{B}^{T} \mathbf{A}_{\kappa}\right\| \leq \alpha \gamma
$$

The derivative of the Lyapunov function is bounded by:

$$
\dot{V} \leq-\lambda_{\min }^{2}\left(\mathbf{A}_{\kappa}\right)\|\eta\|^{2}+\left(\nu \sqrt{N}\left(1+d_{\max }\right)+\alpha \gamma\right)\|\eta\|
$$

Based on the proof of Proposition 2 from [2] a closed ball $B_{\beta}$ centered at $\eta=0$ is defined with radius

$$
\beta=\frac{\nu \sqrt{N}\left(1+d_{\max }\right)+\alpha \gamma}{\lambda_{\min }^{2}\left(\mathbf{A}_{\kappa}\right)}
$$

Let $\Omega_{m}=\{\eta: V(\eta) \leq m\}$ be a level set of the Lyapunov function $V(\eta)$ with $\bar{m}=\frac{1}{2} \lambda_{\max }\left(\mathbf{A}_{\kappa}\right) \beta^{2}$. Then, $B_{\beta}$ is contained in $\Omega_{c}$ because

$$
\|\eta\| \leq \beta \Longrightarrow V(\eta)=\frac{1}{2} \eta^{T} \mathbf{A}_{\kappa} \eta \leq \frac{1}{2} \lambda_{\max }\left(\mathbf{A}_{\kappa}\right) \beta^{2}=m,
$$

and thus $\eta \in \Omega_{m}$. As a result, any solution of (9) starting in $\mathbb{R}^{2 N} \backslash \Omega_{m}$ satisfies $\dot{V}<0$. Thus, it enters $\Omega_{m}$ in some finite time and remains in $\Omega_{m}$ thereafter. This guarantees global asymptotic $\epsilon$-stability of $\eta=0$ with a radius $\epsilon=$ $\beta \lambda_{\max }\left(\mathbf{A}_{\kappa}\right) / \lambda_{\min }\left(\mathbf{A}_{\kappa}\right)$. To show this, note that

$$
\frac{1}{2} \lambda_{\min }\left(\mathbf{A}_{\kappa}\right)\|\eta\|^{2} \leq V(\eta) \leq \frac{1}{2} \lambda_{\max }\left(\mathbf{A}_{\kappa}\right) \beta^{2}
$$

Thus, the solutions enter the region

$$
\|\eta\| \leq \beta \sqrt{\frac{\lambda_{\max }\left(\mathbf{A}_{\kappa}\right)}{\lambda_{\min }\left(\mathbf{A}_{\kappa}\right)}}
$$

which implies the radius of $\epsilon$-stability is

$$
\epsilon=\frac{\nu \sqrt{N}\left(1+d_{\max }\right)+\alpha \gamma}{\lambda_{\min }^{2}\left(\mathbf{A}_{\kappa}\right)} \sqrt{\frac{\lambda_{\max }\left(\mathbf{A}_{\kappa}\right)}{\lambda_{\min }\left(\mathbf{A}_{\kappa}\right)}}
$$

The $\epsilon$-stability of $\eta=0$ implies $\epsilon$-tracking of the mean vector $\mathbf{u}^{*}$ by every agent, therefore $\epsilon$-consensus is asymptotically reached.

After the previous detailed analysis this result can be presented as a theorem:

Theorem 1 Consider a circular formation of $N$ agents defined by (3) with a connected communication graph $\mathcal{G}$ and Assumption 1 is satisfied. Let $\rho: \mathbb{R}^{2} \rightarrow \mathbb{R}^{+}$be a bounded function and the mean vector $u^{*}$ defined in (7) satisfies $\left\|\dot{u}^{*}\right\| \leq \nu$. Then, $z^{*}(t)=1 \otimes u^{*}$ is a globally asymptotically $\epsilon$-stable equilibrium of the dynamics of the distributed algorithm given by

$$
\begin{array}{r}
\dot{\boldsymbol{z}}=-\kappa \boldsymbol{L}_{2} \boldsymbol{z}-\boldsymbol{L}_{2} \boldsymbol{u}+\left(\boldsymbol{I}_{N}+\Delta\right)_{2}(\boldsymbol{u}-\boldsymbol{z}) \\
\text { with } \boldsymbol{u}=\left(\rho_{1}\left(r_{1}-c\right)^{T}, \ldots, \rho_{N}\left(r_{N}-c\right)^{T}\right)^{T} \text { and } \\
\epsilon=\frac{\left(\nu \sqrt{N}\left(1+d_{\max }\right)+\alpha \gamma\right) \lambda_{\max }^{\frac{1}{2}}\left(\boldsymbol{A}_{\kappa}\right)}{\lambda_{\text {min }}^{\frac{5}{2}}\left(\boldsymbol{A}_{\kappa}\right)}
\end{array}
$$




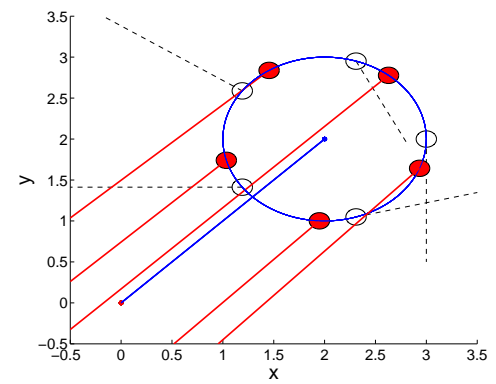

(a) Estimated directions $z_{k}$ for $t=0 \mathrm{~s}$ (black dashed lines) and for $t=50 \mathrm{~s}$ (red lines)
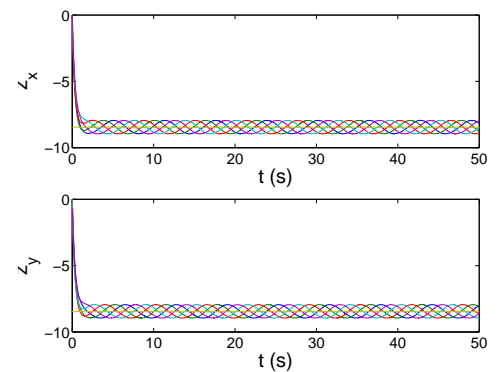

(b) Components of estimated directions $z_{k}$

Fig. 2. Simulation of a circular formation of five agents centered at $c=$ $(2,2)^{T}$. The function $\rho$ representing the signal distribution centered at the origin has circular level sets. The consensus algorithm of Theorem 1 is implemented with $\kappa=50$.

where the matrix $\boldsymbol{A}_{\kappa}$ and the constants $\alpha$ and $\gamma$ are previously defined.

Remark 1 Analyzing the linear system (8) it seems evident that the control parameter $\kappa$ has an important role in the convergence velocity of the algorithm. The simulation results show that taking $\kappa>>1$ the amplitude of oscillations of the estimated gradient directions $z_{k}$ are smaller. Therefore, the error $\eta$ is also reduced. However, the analysis of this property is still under investigation.

\section{B. Simulations}

In this section we present simulations results of the previous algorithm. All simulations show a circular formation of five agents with radius $R=1 \mathrm{~m}$ and angular velocity of $\omega_{0}=1 \mathrm{rad} / \mathrm{s}$. The communication graph is a ring.

In Figs. 2 and 3, the source-seeking consensus algorithm (9) from Theorem 1 is implemented with $\kappa=50$. For these simulations, the function $\rho$ representing the signal distribution centered at the origin has circular level sets, $\rho(x, y)=100 e^{-\left(x^{2}+y^{2}\right) / 10}$. Therefore, the gradient vector $\nabla \rho(c)$ provides the adequate direction to steer the formation to the source location. Both figures (a) show two snapshots. The void circles represent the initial conditions and the black dashed lines the initial estimated direction $z_{k}$ of each agent. The red circles represent the position of the agents at $t=50 \mathrm{~s}$ and the red lines are the estimated gradient

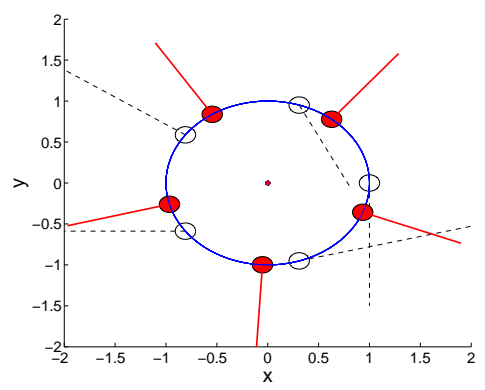

(a) Estimated directions $z_{k}$ for $t=0 \mathrm{~s}$ (black dashed lines) and for $t=50 \mathrm{~s}$ (red lines)
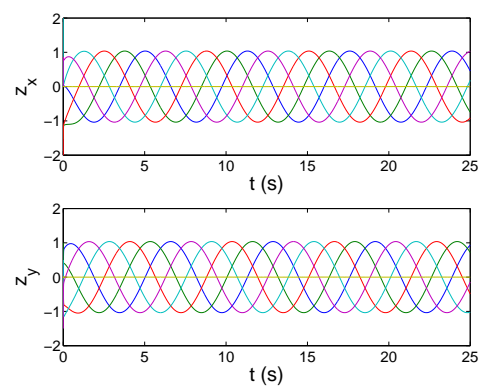

(b) Components of estimated directions $z_{k}$

Fig. 3. Simulation of a circular formation of five agents centered at $c=$ $(0,0)^{T}$. The function $\rho$ representing the signal distribution centered at the origin has circular level sets. The consensus algorithm (9) is implemented with $\kappa=50$.

directions at that time. The blue line is the real direction of the gradient at center $c$. Both figures (b) show the components of the consensus variable $z_{k}$ and the mean vector $u^{*}$. The estimated directions $z_{k}$ oscillate around the vector $u^{*}$ which approximates the true gradient direction for any initial conditions.

In Fig. 2 the circular formation is centered at $c=(2,2)$ and the oscillations of the estimated gradient directions $z_{k}$ are smaller than in Fig. 3 where the formation is centered at source location. In this second case, as the mean of the directions is equal to zero, the convergence region of radius $\epsilon$ leads to completely wrong gradient direction estimations.

\section{Conclusions and limitations of the algorithm}

The final gradient direction $z_{k}$ estimated by each agent oscillates with period $T=2 \pi / \omega_{0}$. The amplitude of these oscillations depends on the concentration measurements $\rho_{k}$. When the formation is closer to the source location, the measurements are greater, thus, the amplitude of oscillations are greater as well. Moreover, as the gradient is closed to zero in the neighborhood of the source (at least with the Gaussian profile we use), a ball of radius $\epsilon$ around 0 leaves the gradient direction essentially unknown; thus Theorem 1 does not guarantee good behavior in the neighborhood of the source.

Another limitation of the previous consensus algorithm (9) is that the radius $\epsilon$ depends on the constants $\alpha$ and $\gamma$ 


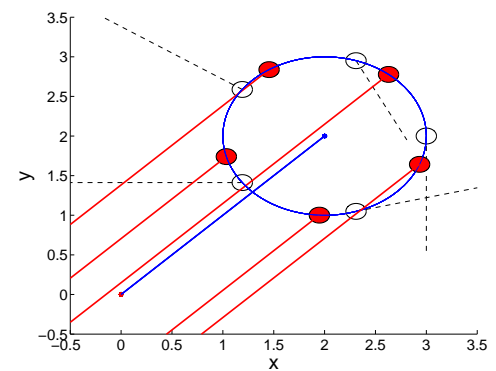

(a) Directions $z_{k}$ for $t=0 \mathrm{~s}$ (black dashed lines) and for $t=50 \mathrm{~s}$ (red lines)
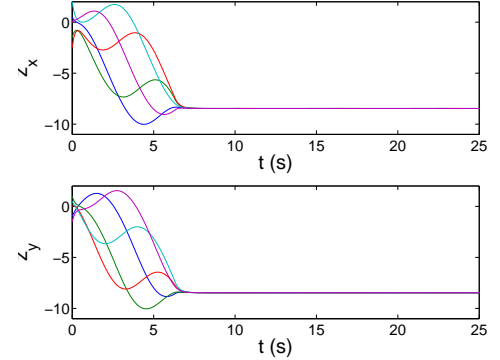

(b) Components of estimated directions $z_{k}$

Fig. 4. Simulation of a circular formation of five agents centered at $c=$ $(2,2)^{T}$. The function $\rho$ representing the source centered at the origin has circular level sets. The mean input consensus algorithm (9) is implemented.

which cannot necessarily be small values. In order to avoid these problems, an averaging approach is presented in the following section.

\section{Refined Collaborative Estimation of GRADIENT DiRECTION RESULT}

The previous section presents a collaborative algorithm, which uses the concentration measurements obtained by a formation of agents to estimate the gradient direction of a signal distribution in its center.

The agents describe a periodic movement, it means that $r_{k}(t)=r_{k}(t+T)$ with $T=2 \pi / \omega_{0}$. Therefore, the measurements $\rho_{k}$ obtained by agent $k$ is a periodic map because $\rho\left(r_{k}(t)\right)=\rho\left(r_{k}(t+T)\right)$. In conclusion, the input variable of the consensus algorithm $u_{k}=\rho_{k}\left(r_{k}-c\right)$ is a $T$ periodic function with $T=2 \pi / \omega_{0}$. Estimated directions $z_{k}$ obtained by the consensus algorithm (9) shown in Figs. 2 and 3 are also periodic. The average of these solutions approximates the gradient direction of the source. Thanks to these observations, an analysis of the average properties of the input variable $u_{k}$ seems adequate. In this section, the previous distributed consensus algorithm is improved using the periodic properties of the measurements $\rho\left(r_{k}\right)$.

\section{A. Estimation Algorithm using time-average inputs}

We present an improved estimation algorithm based on the periodic properties of the input $u_{k}$. The input vector $u_{k}$ in previous consensus algorithm is replaced by its mean value

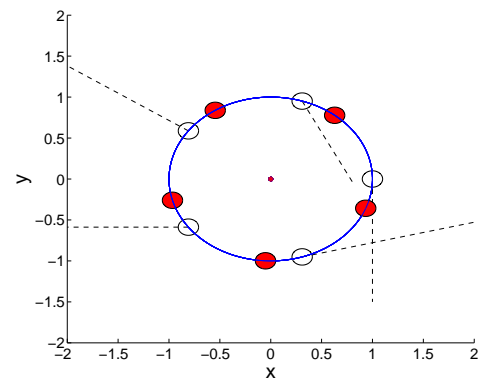

(a) Directions $z_{k}$ for $t=0 s$ (black dashed lines) and for $t=50 \mathrm{~s}$ (red lines)
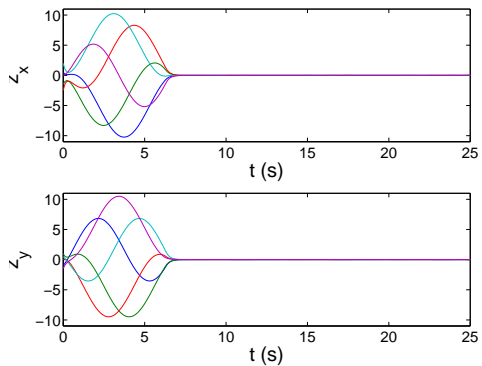

(b) Components of estimated directions $z_{k}$

Fig. 5. Simulation of a circular formation of five agents centered at $c=(0,0)^{T}$. The function $\rho$ representing the source centered at the origin has circular level sets. The mean input consensus algorithm of (12) is implemented.

over one period $T=2 \pi / \omega_{0}$ which is defined as:

$$
\bar{u}_{k}=\frac{1}{T} \int_{t-T}^{t} \rho_{k}\left(r_{k}(\tau)-c\right) d \tau
$$

Therefore, thanks to Lemma 1 (ii) the new mean vector $\bar{u}^{*}$ approximates the gradient of the signal propagation $\rho$ in the center of the circular formation:

$$
\bar{u}^{*}=\frac{1}{N} \sum_{k=1}^{N} \bar{u}_{k}
$$

The new input variable of the improved algorithm based on (9), is the mean vector $\overline{\mathbf{u}}=\left(\bar{u}_{1}^{T}, \bar{u}_{2}^{T}, \ldots, \bar{u}_{N}^{T}\right)^{T}$, and the objective is defined as $\overline{\mathbf{u}}^{*}=\mathbf{1} \otimes \bar{u}^{*}$. Following the analysis developed in section IV-A, let us assume that the following inequality $\left\|\left(\overline{\mathbf{u}}-\overline{\mathbf{u}}^{*}\right)\right\| \leq \bar{\alpha}$ is satisfied. Using these considerations, a new algorithm is proposed in the following corollary:

Corollary 1 Consider a circular formation of $N$ agents defined by (3) with a connected communication graph $\mathcal{G}$ and Assumption 1 is satisfied. Let $\rho: \mathbb{R}^{2} \rightarrow \mathbb{R}^{+}$be a bounded function and the mean vector $\bar{u}^{*}$ defined in (11) satisfies $\left\|\dot{\bar{u}}^{*}\right\| \leq \bar{\nu}$. Then, $z^{*}(t)=\mathbf{1} \otimes \bar{u}^{*}$ is a globally asymptotically $\bar{\epsilon}$-stable equilibrium of the dynamics of the distributed algorithm given by

$$
\dot{z}=-\kappa \boldsymbol{L}_{2} \boldsymbol{z}-\boldsymbol{L}_{2} \overline{\boldsymbol{u}}+\left(\boldsymbol{I}_{N}+\Delta\right)_{2}(\overline{\boldsymbol{u}}-\boldsymbol{z})
$$


with

$$
\bar{\epsilon}=\frac{\left(\bar{\nu} \sqrt{N}\left(1+d_{\max }\right)+\bar{\alpha} \gamma\right) \lambda_{\max }^{\frac{1}{2}}\left(\boldsymbol{A}_{\kappa}\right)}{\lambda_{\min }^{\frac{5}{2}}\left(\boldsymbol{A}_{\kappa}\right)}
$$

Remark 2 Considering Assumption 1 (the circular formation is fixed) by definition, the mean input $\overline{\boldsymbol{u}}$ is a constant vector after a time period $T$. Therefore, the input variable $\overline{\boldsymbol{u}}$ converges to the mean vector $\overline{\boldsymbol{u}}^{*}$ and moreover, its derivative is equal to zero. Then, $\bar{\nu} \rightarrow 0$ and $\bar{\alpha} \rightarrow 0$. It implies that the radius of the convergence region $\bar{\epsilon}$ converges to zero after a period $T$, the consensus is achieved and all the agents estimate the mean vector $\bar{u}^{*}$ which approximates the gradient direction at the center of the formation. Moreover, thanks to this result, the parameter $\kappa$ has less influence in the improved algorithm, thus we can consider $\kappa=1$.

Remark 3 The gradient direction estimated by the agents will be the velocity reference of the formation center to steer the fleet of agents to the source location. If the formation is moving, the gradient of the signal distribution in the circle center is also moving and the concentration measurements does not satisfy the periodic properties anymore. Therefore, the consensus algorithm makes that the agents would estimate the gradient direction before a period T. A detailed investigation of our algorithms when the formation moves along the estimated gradient direction towards the source location is our next research goal.

\section{B. Simulations}

The simulations show the same circular formation of five agents from the previous simulations. In Figs. 4 and 5 the improved distributed algorithm (12) from Corollary 1 is implemented with $\kappa=1$ by a circular formation centered at $c=(2,2)^{T}$ and at source location, respectively. The measured signal is the same as in previous simulations. Due to the circular level sets of the signal propagation the gradient vector $\nabla \rho(c)$ provides the adequate direction to steer the formation to the source location. Both figures (a) show two snapshots, the initial conditions and the stable situation at $t=50 \mathrm{~s}$. Both figures (b) show the components of consensus variable $z_{k}$. This algorithm allows to remove the oscillations and the final vectors $z_{k}$ (red lines) are parallel to the gradient direction for all the agents (blue line). The problem of oscillations when the formation is centered at source location is also solved and the final directions $z_{k}$ are equal to zero, i.e., the formation decides to stay in the desired location. The estimated directions $z_{k}$ converge to the gradient direction approximated by the mean vector $\bar{u}^{*}$ for any initial conditions.

In Fig. 6 the same algorithm (12) is implemented with an elliptical signal distribution defined by $\rho(x, y)=$ $100 e^{-\left(x^{2} / 10+y^{2} / 2\right) / 10}$. The estimated directions $z_{k}$ converge to the gradient direction $\nabla \rho(c)$. In this case, this direction will not directly steer the formation to the source location, but a formation moving along the respective gradient direc-

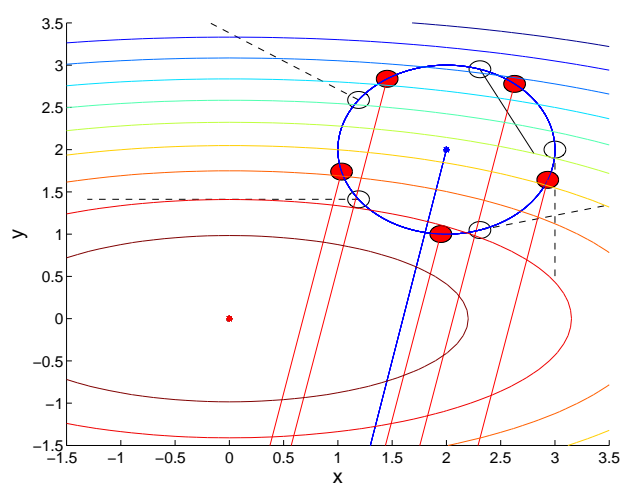

Fig. 6. Simulation of the distributed algorithm (12) by a circular formation of five agents centered at $c=(2,2)^{T}$. The function $\rho$ representing the source centered at the origin has elliptical level sets.

tion will be progressively steered towards the source over several consecutive steps.

\section{CONCLUSIONS}

This paper presents a cooperative multi-agent algorithm to estimate the gradient direction of a signal distribution. This distributed algorithm uses the concentration measurements of the signal obtained by a group of vehicles uniformly distributed in a fixed circular formation. Our approach takes into account the communication constraints of the network, avoiding the case of all-to-all communication. To achieve this objective, a first distributed consensus algorithm based on instantaneous sensor measurements is presented. Then, we propose an improved algorithm based on the average inputs. We note that if the formation is fixed, this second algorithm reaches exact consensus and converge to the gradient direction at the formation center.

This analysis requires that formation is fixed. Our final objective is to use this estimated direction to locate the source. Then, our next research aim is to analyze and improve these algorithms when the formation moves along the estimated gradient direction towards the source location.

\section{REFERENCES}

[1] R. Olfati-Saber, J. A. Fax, and R. M. Murray, "Consensus and cooperation in networked multi-agent systems," Proceedings of the IEEE, vol. 95, pp. 215-233, 2007.

[2] R. Olfati-Saber and J. S. Shamma, "Consensus filters for sensor networks and distributed sensor fusion," in Proceedings of the 44th IEEE Conference on Decision and Control, and the European Control Conference 2005, Seville, Spain, 2005, pp. 6698-6703.

[3] R. Olfati-Saber, "Flocking for multi-agent dynamic systems: Algorithms and theory," IEEE Trans. on Automatic Control, vol. 51, pp. 401-420, 2006.

[4] P. Ögren, E. Fiorelli, and N. E. Leonard, "Cooperative control of mobile sensor networks: Adaptive gradient climbing in a distributed environment," IEEE Transactions on Automatic Control, vol. 49, pp. 1292-1302, 2004.

[5] F. Zhang and N. E. Leonard, "Cooperative filters and control for cooperative exploration," IEEE Trans. on Automatic Control, vol. 55, pp. 650-663, 2010.

[6] N. E. Leonard, D. A. Paley, F. Lekien, R. Sepulchre, D. M. Frantatoni, and R. E. Davis, "Collective motion, sensor networks and ocean sampling," Proceedings of the IEEE, vol. 95, pp. 48-74, 2007. 
[7] R. L. Raffard, C. J. Tomlin, and S. P. Boyd, "Distributed optimization for cooperative agents: Application to formation flight," in Proceedings of 43rd IEEE Conference on Decision and Control, Paradise Islands, The Bahamas, vol. 3, 2004, pp. 2453-2459.

[8] J. A. Fax and R. M. Murray, "Information flow and cooperative control of vehicle formations," IEEE Transactions on Automatic Control, vol. 49, pp. 1465-1476, 2004.

[9] A. Sarlette, S. Bonnabel, and R. Sepulchre, "Coordinated motion design on Lie groups," IEEE Transactions on Automatic Control, vol. 55, no. 5, pp. 1047-1058, 2010.

[10] S. Martínez, J. Cortés, and F. Bullo, "Motion coordination with distributed information," IEEE Control Systems Magazine, vol. 27, pp. 75-88, 2007.

[11] P. Ögren, M. Egerstedt, and X. Hu, "A control lyapunov function approach to multiagent coordination," IEEE Transactions on Automatic Control, vol. 18, pp. 847-851, 2002.

[12] R. Sepulchre, D. A. Paley, and N. E. Leonard, "Stabilization of planar collective motion with limited communication," IEEE Transactions on Automatic Control, vol. 53, pp. 706-719, 2008.

[13] L. Scardovi, N. E. Leonard, and R. Sepulchre, "Stabilization of collective motion in three dimensions," Communications in Information and Systems (Brockett Legacy issue), vol. 8, pp. 1521-1540, 2008.

[14] S. Hernandez and D. A. Paley, "Three-dimensional motion coordination in a time-invariant flowfield," in Proceedings of the 48th IEEE Conference on Decision and Control held jointly with the 28th Chinese Control Conference, Shanghai, China, 2009, pp. 7043-7048.

[15] D. A. Paley and C. Peterson, "Stabilization of collective motion in a time-invariant flowfield," Journal of Guidance, Control, and Dynamics, vol. 32, pp. 771-779, 2009.

[16] D. A. Paley, N. E. Leonard, and R. Sepulchre, "Stabilization of symmetric formations to motion around convex loops," Systems \& Control Letters, vol. 57, pp. 209-215, 2008.

[17] L. Briñón-Arranz, A. Seuret, and C. Canudas-de-Wit, "Elastic formation control based on affine transformations," in Proceedings of the 2011 American Control Conference, San Francisco, USA, 2011, pp. 3984-3989.

[18] — , "Translation control of a fleet circular formation of AUVs under finite communication range," in Proceedings of the 48th IEEE Conference on Decision and Control held jointly with the 28th Chinese Control Conference, Shanghai, China, 2009, pp. 8345-8350.

[19] M. Krstić and H.-H. Wang, "Stability of extremum seeking feedback for general nonlinear dynamic systems," Automatica, vol. 36, pp. 595$601,2000$.

[20] J. Cochran and M. Krstić, "Source seeking with a nonholonomic unicycle without position measurements and with tuning of angular velocity Part I: Stability analysis," in Proceedings of 46th IEEE Conference on Decision and Control, New Orleans, LA, USA, 2007, pp. 6009-6016.

[21] S. S. Sahyoun, S. M. Djouadi, and H. Qi, "Dynamic plume tracking using mobile sensors," in Proceedings of the 2010 American Control Conference, Baltimore, MD, USA, 2010, pp. 2915-2920.

[22] M. Rabbat and R. Nowak, "Distributed optimization in sensor networks," in Proceedings of the 3rd International symposium on Information processing in sensor networks, Berkeley, CA, USA, 2004, pp. 20-27.

[23] B. J. Moore and C. Canudas-de-Wit, "Source seeking via collaborative measurements by a circular formation of agents," in Prooceedings of the 2010 IEEE American Control Conference, Baltimore, USA, 2010, pp. 6417-6422. 